J. Austral. Math. Soc. 20 (Series A) (1975), 54-58.

\title{
A NOTE ON MATROIDS AND BLOCK DESIGNS
}

\author{
R. A. MAIN and D. J. A. WELSH
}

(Received 28 May 1973)

Communicated by G. Szekeres

\section{Introduction}

The close connection between certain types of matroids or combinatorial geometries and block designs is well known. The relationships previously discussed have centred on the loose analogy between the blocks of a design and the hyperplanes or flats ot the matroid or geometry. The matroids whic arise in this way have had in the main a very tight regular structure. Here we show that the class of matroids whose bases are the blocks of a design is much wider - indeed from Theorem 6 below we obtain a matroid in a canonical way from any balanced incomplete block design in which no pair of blocks differ by exactly one element.

The block design terminology is standard, being that of the book by Hall (1967) and unless otherwise specified the general balanced incomplete block design or BIBD will be assumed to have parameters $b, v, r, k, \lambda$. We will also only be concerned with designs in which any two blocks are distinct as sets.

The complementary design $D^{*}$ of a design $D$ on a set $S$ has as its blocks the sets $\left\{S \backslash B_{i}: B_{i}\right.$ a block of $\left.D\right\}$. The subtract $\bar{D}$ of a $(b, v, r, k, \lambda)$-design $D$ is the design whose blocks are those $k$-sets which are not blocks of $D$. It is obvious that $\bar{D}$ is a BIBD; we have been unable to find a name for it in the literature and have accordingly called it the subtract. A Steiner system $S(d, k, n)$ is a collection of $k$-sets (blocks) of an $n$-set $S$ such that each $d$-set in $S$ is contained in a unique block.

The matroid terminology is also very standard - see for example Harary and Welsh (1969). The following classes of matroid have previously been encountered in the literature.

A matroid $\mathscr{M}$ is equicardinal if all its hyperplanes have the same cardinality. A matroid design is a matroid whose hyperplanes are the blocks of a design. A MYED (named after its founders Young, Murty, Edmonds (1970) is a matroid $\mathscr{M}$ in which every $k$-flat has the same cardinal for $1 \leqq k \leqq r(\mathscr{M})$ where $r$ is the rank function. They show that a MYED is a matroid design but not conversely. 


\section{Base Designs}

A base design is a collection $\mathscr{B}$ of distinct subsets $\left\{B_{i}: 1 \leqq i \leqq b\right\}$ of a finite set $S$ such that

(1) $\mathscr{B}$ is the set of bases of a matroid $\mathscr{M}(\mathscr{B})$ on $S$

(2) $\mathscr{B}$ is the set of blocks of BIBD $D(\mathscr{B})$ on $S$.

\section{Examples}

1. If $\mathscr{B}$ is the set of all $k$-sets of $S$, then $\mathscr{B}$ is a base design. We call such a design trivial.

2. Ingleton (1971) pointed out that the basis of a projective geometry $P G(n, q)$ form a base design. Similarly the bases of an affine geometry $A G(n, q)$ form a base sdeign.

3. Young, Murty and Edmonds (1970) show that if $\mathscr{M}$ is a MYED and $\mathscr{J}_{k}$ is the set of independent sets of cardinals $k$ then $\mathscr{J}_{k}$ is a base design. This includes the class 2 , since all projective and affine geometries are MYEDs.

We now present some properties of base designs which enable us to show that the class of such designs is much wider than we had originally expected.

It is easy to prove that a matroid design must be connected. We now obtain the analogous result for base design.

THEOREM 1. If $\mathscr{B}$ is a base design then the matroid $\mathscr{M}(\mathscr{B})$ is connected.

Proof. Suppose $\mathscr{M}=\mathscr{M}(\mathscr{B})$ is disconnected with components $\mathscr{M}_{1}$ on $S_{1}, \mathscr{M}_{2}$ on $S_{2}, S_{1} \cap S_{2}=\varnothing$. Any base $B$ of $\mathscr{M}$ is of the form $B_{1} \cup B_{2}$, where $B_{i}$ is a base of $\mathscr{M}_{i}$.

If $S_{i}$ is a single element $\{x\}$ then either $x$ belongs to every base of $\mathscr{M}$, or $x$ belongs to no base of $\mathscr{M}$, impossible.

Hence $\left|S_{i}\right| \geqq 2$. Let $x_{1}, y_{1}$ be distinct elements of $S_{1}, x_{2}, y_{2}$ distinct elements of $S_{2}$. Let $\left\{x_{1}, y_{1}\right\}$ be contained in $\lambda_{1}$ bases of $\mathscr{M}_{1},\left\{x_{2}, y_{2}\right\}$ in $\lambda_{2}$ bases of $\mathscr{M}_{2}$ and let $\mathscr{M}_{1}, \mathscr{M}_{2}$ have respectively $b_{1}, b_{2}$ bases.

Then $\left\{x_{1}, y_{1}\right\}$ is contained in $\lambda_{1} b_{2}$ bases of $\mathscr{M}$ and $\left\{x_{2}, y_{2}\right\}$ is in $\lambda_{2} b_{1}$ bases of $\mathscr{M}$. Thus

$$
\lambda_{1} b_{2}=\lambda_{2} b_{1}=\lambda
$$

Also if $x_{1}$ is in $r_{1}$ bases of $\mathscr{M}_{1}, x_{2}$ in $r_{2}$ bases of $\mathscr{M}_{2}, x_{1}$ is in $r_{1} b_{2}$ bases of $\mathscr{M}, x_{2}$ is in $r_{2} b_{1}$ bases of $\mathscr{M}$, hence

$$
r_{1} b_{2}=r_{2} b_{1}=r
$$

Now $x_{1} \neq x_{2}$, hence $\left\{x_{1}, x_{2}\right\}$ is contained in $\lambda$ bases of $\mathscr{M}$

$$
\lambda=r_{1} r_{2}
$$


Simple manipulation of (1), (2) and (3) together with the facts $b k=v r, \lambda(v-1)$ $=r(k-1), b=b_{1} b_{2}$ give the conclusion that $v=k$, which means that the design does not exist.

THEOREM 2. If $\mathscr{B}$ is a base design then the complementary design is also a base design and is in fact the set of bases of the dual matroid $[\mathscr{M}(\mathscr{B})]^{*}$.

Our next result shows that there are essentially no symmetric base designs.

THEOREM 3. The only base design $\mathscr{B}$ such that $D(\mathscr{B})$ is a symmetric design is the trivial design where $\mathscr{B}$ is the set of $(n-1)$-sets of an $n$-set.

Proof. Since $\mathscr{B}$ is the collection of bases of a matroid, by the exchange axiom there exist bases $B_{1}, B_{2}$ such that $\left|B_{1}\right| B_{2} \mid=1$. Hence $\left|B_{1} \cap B_{2}\right|$ $=\left|B_{1}\right|-1$. But in a symmetric design any two blocks intersect in $\lambda$ elements. Thus $\mathscr{B}$ must be a trivial $(k+1, k, k-1)$ design.

THEOREM 4. Necessary conditions that the blocks of $a(b, v, r, k, \lambda)$ design be a base design are that, except when the design is trivial,

(i) $\lambda \geqq k-1 \Leftrightarrow r \geqq v-1$

(ii) $(r-1)(v-k) \geqq b-1 \geqq r k /(k-1)$,

(iii) $b-r \geqq v-1$.

Proof. (i) Take any block $B$ of the design, $B=\left\{b_{1}, b_{2} \cdots, b_{k}\right\}$. Since there are at least $v$ blocks we know that for any $e \in S$, there is a block not containing $e$. Take $B_{i}$ to be a base not containing $b_{i}$. Then by the exchange axiom $\exists f \in B_{i}$ such that $B_{i}{ }^{\prime}=\left(B \backslash\left\{b_{i}\right\}\right) \cup\{f\}$ is a base and hence a block of $D$. Now $B_{i}^{\prime} \neq B_{j}^{\prime}$ for $i \neq j$ since $b_{i} \in B_{j}^{\prime}$ and $b_{i} \notin B_{i}^{\prime}$. Consider the set of blocks $\left\{B, B_{1}^{\prime}, B_{2}^{\prime}, \cdots, B_{k}^{\prime}\right\}$ : for $i \neq j,\left\{b_{i}, b_{j}\right\}$ is contained in exactly $k-1$ blocks of this set. Hence $\lambda \geqq k-1$.

(ii) This result seems to demand a great deal of work and so fari has not proved to be of any use, we therefore omit the proof.

(iii) Apply the same argument as in (i) to the dual matroid by theorem 2 .

We next show that a large class of base designs can be derived from matroid designs.

THEOREM 5. If $D$ is a matroid design then the blocks of the subtract $\bar{D}$ of $D$ form a base design.

It is easy to see that theorem 5 is not true if $D$ is just an equicardinal matroid.

Nor is the converse true; the subtract of the base design $P G(2,3)$ is not a matroid design.

Proof. Let $B_{1}, B_{2}$ be distinct blocks of $\bar{D}$, let'e $\in B_{1} \mid B_{2}$. Suppose there does not exist $f \in B_{2} \mid B_{1}$ such that $\left(B_{1} \mid\{e\}\right) \cup\{f\}$ is a block of $\bar{D}$. Then $\forall f \in B_{2} \mid B_{1}$, $\left(B_{1} \mid\{e\}\right) \cup\{f\}$ is a block of $D$.

Suppose $B_{2} \mid B_{1}=\{f\}$, then $\left(B_{1} \mid\{e\}\right) \cup\{f\}=B_{2}$ is a block of $\bar{D}$. 
Hence assume $B_{2} \mid B_{1}$ contains at least two distinct elements $f_{1}, f_{2}$. Then $\left(B_{1} \mid\{e\}\right) \cup\left\{f_{1}\right\}$ and $\left(B_{1} \mid\{e\}\right) \cup\left\{f_{2}\right\}$ are both blocks of $D$. Since $D$ is a matroid design

$$
\left(\left(B_{1} \mid\{e\}\right) \cup\left\{f_{1}\right\}\right) \cap\left(\left(B_{1} \mid\{e\}\right) \cup\left\{f_{2}\right\}\right) \cup\{e\}=B_{1}
$$

must be contained in a block of $D$, and hence must be a block of $D$, which is impossible. Thus $\bar{D}$ is a base design.

A much wider class of base designs is obtained from the following result.

THEOREM 6. If $D$ is $a(b, v, r, k, \lambda) B I B D$ in which no two distinct blocks intersect in a set of cardinal $k-1$ then the subtract of $D$ is the set of blocks of a base design.

Note: it is easy to see that a MYED $D$ has a pair of blocks $B_{i}, B_{j}$ such that

$$
\left|B_{i} \cap B_{j}\right|=\left|B_{i}\right|-1
$$

if and only if $D$ is trivial. Hence Theorem 6 includes that part of Theorem 5 which refers to MYEDS. However if one considers the Steiner system $S(5,8,24)$, its blocks are a MYED and its circuits are those 6-sets which are contained in some block. Hence there exist circuits $C_{i}, C_{j}$ with $\left|C_{i} \cup C_{j}\right|=7$, and thus the complementary design $\left\{S-C_{i}: C_{i}\right.$ a circuit of $\left.S(5,8,24)\right\}$ is a matroid design possessing property (4). Thus Theorem 6 does not include Theorem 5 .

Proof. Let $B_{1}, B_{2}$ be two blocks of the subtract $\bar{D}$ of $D$. Let $e \in B_{1} \mid B_{2}$. If $\nexists f \in B_{2} \mid B_{1}$ with $\left(B_{1} \mid\{e\}\right) \cup\{f\}$ a block of $\bar{D},\left(B_{1} \mid\{e\}\right) \cup\{f\}$ is a block of $D$ $\forall f \in B_{2} \mid B_{1}$. Assuming $\left|B_{2}\right| B_{1} \mid \neq 1$ (in which case the problem is trivial) we now have two blocks of $D$ which intersect in a $(k-1)$-set, contradicting the hypothesis.

\section{Conclusion}

We close by asking the following questions.

1. Is the collection of bases of a matroid design a base design? We think not.

2. If $\mathscr{B}$ is a base design, is the set of bases of any truncation of the associated matroid $\mathscr{M}(\mathscr{B})$ also a base design?

3. It is clear that there exist binary and indeed graphic base designs. The Fano matroid is a base design, the cycle matroid of a tree or a simple cycle is also a base design. However we know of no other graph with this property, and conjecture that the only base designs, which as matroids are graphic, are uniform; that is, are either trees or cycles.

\section{References}

M. Hall (1967), Combinatorial Theory. (Blaisdell, Waltham, 1967).

F. Harary and D. J. A. Welsh (1969), Matroids versus graphs, The Many Facets of Graph Theory, (Springer Lecture Notes), 110, 155-170. 
A. W. Ingleton (1971), (private communication).

U. S. R. Murty (1970), Equicardinal matroids and finite geometries, Combinatorial Structures and their Applications (Gordon and Breach), 289-293.

D. J. A. Welsh (1971), Matroids and block designs Théorie des Matroides, (Springer Lecture Notes) 211, 95-106.

H. Whitney (1935), 'On the abstract properties of linear dependence', Amer. J. Math. 57, 509-533.

P. Young, U. S. R. Murty, and J. Edmonds (1970), Equicardinal matroids and matroid designs, Combinatorial Mathematics and its Applications (Charel Hill), 498-542.

Mathematical Institute

University of Oxford

Oxford, U. K.

Note added in Proof 23.9.74.

W. T. Tutte has recently proved conjecture 3 above, in "A problem on spanning trees", Quart. J. Math. (Oxford) (to appear). 